\title{
MicroRNA-634 alters nerve apoptosis via the PI3K/Akt pathway in cerebral infarction
}

\author{
YAJUN CHANG, WEI HUANG, QIAN SUN, SULI LI, ZHONGKAI YAN, QIUMIN WANG and XIUMIN LIU \\ Department of Biochemistry and Molecular Biology, Tangshan Worker Hospital, Tangshan, Hebei 063000, P.R. China
}

Received January 2, 2018; Accepted May 18, 2018

DOI: $10.3892 / \mathrm{ijmm} .2018 .3777$

\begin{abstract}
In the present study, the role and mechanism of microRNA-634 (miRNA-634) in the adjustment of nerve inflammation and apoptosis in cerebral infarction were investigated. In a cerebral infarction rat model, the expression of miRNA-634 was increased, compared with that in the normal control group. The upregulated expression of miRNA-634 in an in vitro model of cerebral infarction increased cell apoptosis and the protein expression of capsase-3/B-cell lymphoma 2 -associated $\mathrm{X}$ protein (Bax) via inactivation of the phosphoinositide 3-kinase (PI3K)/Akt pathway. The downregulation of miRNA-634 enhanced cell growth and inhibited cell apoptosis in the in vitro model of cerebral infarction through induction of the PI3K/Akt pathway. Subsequently, a PI3K inhibitor was used to inhibit the expression of PI3K in the in vitro model of cerebral infarction via the downregulation of miRNA-634, which showed that cell apoptosis and the protein expression of capsase-3/Bax were also increased. A PI3K agonist reduced the effects of the upregulation of miRNA-634 in the in vitro model of cerebral infarction. In conclusion, the data obtained demonstrated the possible future use of miRNA-634 as a therapeutic target in cerebral infarction through the PI3K/Akt pathway.
\end{abstract}

\section{Introduction}

Cerebral thrombosis is an ischemic cerebrovascular disease (1). It is an important component type of atherosclerosis, accounting for $70-80 \%$ of all patients with stroke. In cerebral thrombosis, $\sim 90 \%$ of cases develop on the basis of cerebral atherosclerosis (2). Therefore, it is frequently referred to as atherosclerotic cerebral thrombosis. Atherosclerosis is a chronic progressive disease. It develops during childhood but clinical symptoms present in middle- and old-aged individuals.

Correspondence to: Mr. Qian Sun, Department of Biochemistry and Molecular Biology, Tangshan Worker Hospital, 27 Wenhua Road, Tangshan, Hebei 063000, P.R. China

E-mail: sxka4009607@126.com

Key words: microRNA-634, cerebral infarction, inflammation, apoptosis, phosphoinositide 3-kinase, Akt
The pathological changes of atherosclerosis mainly involve the aorta and medium-sized elastic arteries (3). Among these, the coronary artery and cerebral arteries are the most dominant (3). Furthermore, the lesion predominantly involves multiple organs at the same time (2). With the increase in detailed investigations on atherosclerosis over the last century, several representative theories have formed successively. These include lipid infiltration, thrombosis, smooth muscle cell clones, injury response and chronic inflammation (4).

MicroRNAs (miRNAs) are a class of small, endogenous, non-coding single-strand RNAs. They are one of the most important gene expression regulatory factors (5). miRNAs interact with the specific sequence of a target gene and inhibits target gene activity or promotes its degradation at the post-transcriptional level (5). In addition, miRNAs regulate target gene expression, and are involved in biological processes that include cell proliferation, differentiation, apoptosis and metabolism (6). The expression of brain-specific miRNAs in the central nervous system is rich, whereas no or minimal expression is observed in the majority of other organs (6). miRNAs are important in nervous system development and function. It is reported that miRNA may be passively leaked out from damaged tissue cells or infiltrated cells, similar to other materials (7). Alternatively, it may be actively secreted from the damaged tissue cells into the blood circulation. The latter is considered to be the major mechanism (6).

The phosphoinositide 3-kinase (PI3K)/Akt signal transduction pathway is an important pathway for the intracellular transduction of membrane receptor signals (8). It is key in maintaining cell survival and inhibiting cell apoptosis. In addition, it can affect the activation process of effector molecules, including downstream apoptosis-related proteins and cell cycle regulating proteins. Therefore, it is crucial in inhibiting apoptosis and promoting proliferation in cells (9). The aim of the present study was to examine the effects of plasma miRNA-634 on adjusting nerve inflammation and apoptosis in cerebral infarction, and investigate its possible mechanism.

\section{Materials and methods}

Middle cerebral artery occlusion (MCAO) model. Male Sprague-Dawley (SD) rats (170-210 g) were purchased for animal experiments from Beijing Vital River Laboratory Animal Technology Co., Ltd. (Beijing, China). All rats 
were housed at $22-23^{\circ} \mathrm{C}$ with a $55-60 \%$ humidity, $12 / 12 \mathrm{~h}$ light/dark cycle and free access to food and water. Cerebral ischemia-reperfusion was induced by MCAO. The SD rats were anesthetized with $35 \mathrm{mg} / \mathrm{kg}$ of pentobarbital (i.p) and randomly assigned into two groups: Control and MCAO model group. A midline neck incision was made and the left common and external carotid arteries was isolated, and was ligated with a microvascular clip (cat. no. FE691, Aesculap, Tuttlingen, Germany). An 8-0 nylon monofilament with silicon resin (180-190 $\mu \mathrm{m}$ ) was introduced through a small incision into the common carotid artery and advanced $9 \mathrm{~mm}$ distal to the carotid bifurcation to induce MCAO. In the control group, the rats were anesthetized with $35 \mathrm{mg} / \mathrm{kg}$ of pentobarbital only without MCAO. After $1 \mathrm{~h}$, reperfusion was initiated by withdrawal of the monofilament. After 1 day, the SD rats were anesthetized with $35 \mathrm{mg} / \mathrm{kg}$ of pentobarbital and sacrificed for subsequent investigations.

$R N A$ extraction and reverse transcription-quantitative polymerase chain reaction (RT-qPCR) analysis. Brain tissue samples were subsequently obtained and hippocampal tissues were isolated. Total RNA from the hippocampal tissues samples was isolated using TRIzol reagent (Invitrogen; Thermo Fisher Scientific, Inc., Waltham, MA, USA). The cDNA was reverse transcribed using the M-MLV Reverse Transcription system (Takara Biotechnology Co., Ltd., Dalian, China). The qPCR analysis was performed using 100 ng cDNA on an Applied Biosystems 7500 Fast Real-Time PCR system with the SYBR-Green PCR kit (TransGen, Beijing, China). The following primers were used for qPCR: miR-634 forward, 5'-CAGTCTCAAACCAGCACC-3' and reverse, 5'-TATGGTTGTTCACGACTCCTTCAC-3'; and U6 forward, 5'-CTCGCTTCGGCAGCACA-3' and reverse, 5'-AACGCTTCACGAATTTGCGT-3'. The qPCR analysis was performed by denaturation at $95^{\circ} \mathrm{C}$ for $10 \mathrm{~min}$, followed by 40 cycles of $95^{\circ} \mathrm{C}$ for $30 \mathrm{sec}, 60^{\circ} \mathrm{C}$ for $30 \mathrm{sec}$ and $72^{\circ} \mathrm{C}$ for $30 \mathrm{sec}$. Relative expression levels of mRNA were calculated by the $2^{-\Delta \Delta \mathrm{Cq}}$ method (10).

Gene expressionmicroarrays.RNA(500 ng) from hippocampal tissues was amplified using fluorescent complementary RNA and a RNA Labeling kit (Arraystar Inc., Rockville, MD, USA) in accordance with the manufacturer's protocol. Array hybridization was performed according to the Agilent One-Color Microarray-Based Gene Expression Analysis protocol (Agilent Technologies, Inc., Santa Clara, CA, USA) and subsequent washing was performed using a mRNA-ONLY ${ }^{\mathrm{TM}}$ Eukaryotic mRNA Isolation kit (Epicentre; Illumina, Inc., San Diego, CA, USA). cDNA samples were labeled with Cy3 using the SureTag DNA Labeling kit (Agilent Technologies, Inc.). Scanning of the microarrays was performed using Feature Extraction software (v.10.7.3.1; Agilent Technologies, Inc.).

Transient transfection of cells. BV2 cells were purchased from the Shanghai Cell Bank of Chinese Academy of Sciences (Shanghai, China) and cultured in Dulbecco's modified Eagle's medium (DMEM; Gibco; Thermo Fisher Scientific, Inc.) with $10 \%$ fetal bovine serum (Gibco; Thermo Fisher Scientific, Inc.), glutamine ( $2 \mathrm{mmol} / \mathrm{l}$, Invitrogen; Thermo Fisher Scientific, Inc.), penicillin (200 U/ml; Hyclone; GE
Healthcare Life Sciences, Logan, UT, USA) and streptomycin (100 $\mu \mathrm{g} / \mathrm{ml}$; Hyclone; GE Health Care Life Sciences) at $37^{\circ} \mathrm{C}$ with $5 \% \quad \mathrm{CO}_{2}$. The miRNA (miR)-634 inhibitor mimics (100 nM; Sangon Biotech Co., Ltd., Shanghai, China) and negative mimics (100 nM; Sangon Biotech Co., Ltd.) were transfected into BV2 cells using Lipofectamine 2000 according to the manufacturer's protocol (Invitrogen; Thermo Fisher Scientific, Inc.). A total of $4 \mathrm{~h}$ post-transfection, BV2 cells were incubated in DMEM medium without glucose or serum in hypoxic conditions $\left(5 \% \mathrm{CO}_{2}\right.$ and $\left.95 \% \mathrm{~N}_{2}\right)$ for $4 \mathrm{~h}$ at $37^{\circ} \mathrm{C}$ followed by incubation under normoxic conditions for $14 \mathrm{~h}$ at $37^{\circ} \mathrm{C}$.

Cell proliferation and cell apoptosis assays. The cells $\left(1 \times 10^{3}\right.$ per well) were grown in $96-w e l l$ plates and analyzed with an MTT assay for $4 \mathrm{~h}$ at $37^{\circ} \mathrm{C}$. DMSO was added to dissolve crystals for $20 \mathrm{~min}$ at $37^{\circ} \mathrm{C}$. The luminescence was then measured using a microplate reader (BioTek Instruments, Inc., Vinooski, VT, USA) at $492 \mathrm{~nm}$.

For the analysis of apoptosis, the cells were proliferated in 6-well plates for $48 \mathrm{~h}$ following transfection, following which the cells were washed once in PBS and resuspended with binding buffer. The cells were stained with $5 \mu \mathrm{l}$ fluorochrome-conjugated Annexin V and $5 \mu \mathrm{l}$ PI (BD Biosciences, Franklin Lakes, NJ, USA). Apoptosis was detected using flow cytometry (FACSCanto; BD Biosciences).

Enzyme-linked immunosorbent assay (ELISA). The cell supernatants were collected and protein content was measured using a BCA assay. The levels of capsase- 3 , capsase- 8 and capsase- 9 were quantified using commercially available ELISA kits.

Western blot analysis. Total protein extracts were obtained from the cultured cells using RIPA assay and centrifuged at $10,000 \mathrm{~g}$ for $5 \mathrm{~min}$ at $4^{\circ} \mathrm{C}$. Protein content was measured using a BCA assay. The protein samples $(50 \mu \mathrm{g})$ were separated with 8-12\% SDS-PAGE and transferred onto nitrocellulose membranes. The membranes were then blocked with 5\% BSA in TBST and incubated overnight at $4^{\circ} \mathrm{C}$ with the following primary antibodies: B-cell lymphoma 2 (Bcl-2)-associated X protein (Bax; cat. no. sc-6236; 1:1,000; Santa Cruz Biotechnology, Inc., Santa Cruz, CA, USA), PI3K (cat. no. sc-1331; 1:1,000; Santa Cruz Biotechnology, Inc.), phosphorylated (p-)Akt (cat. no. sc-7985-R; 1:1,000; Santa Cruz Biotechnology, Inc.), MDM2 proto-oncogene (MDM2; cat. no. sc-812; 1:1,000; Santa Cruz Biotechnology, Inc.), p53 (cat. no. sc-47698; 1:1,000; Santa Cruz Biotechnology, Inc.) and GAPDH (cat. no. sc-293335; 1:2,000; Santa Cruz Biotechnology, Inc.). The membranes were then washed with TBST and incubated for $1 \mathrm{~h}$ at $37^{\circ} \mathrm{C}$ with HRP-conjugated goat anti-rabbit IgG (cat. no. sc-2004; 1:5,000; Santa Cruz Biotechnology, Inc.) or HRP-conjugated goat anti-mouse IgG secondary antibodies (cat. no. sc-2005; 1:5,000; Santa Cruz Biotechnology, Inc.). The protein bands were visualized using ECL western blotting detection reagents (Thermo Fisher Scientific, Inc.).

Statistical analysis. All data are expressed as the mean \pm standard error of the mean using SPSS software (version 21.0; IBM Corp., Armonk, NY, USA). All statistical 
A

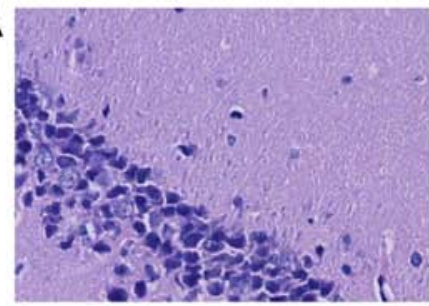

Normal

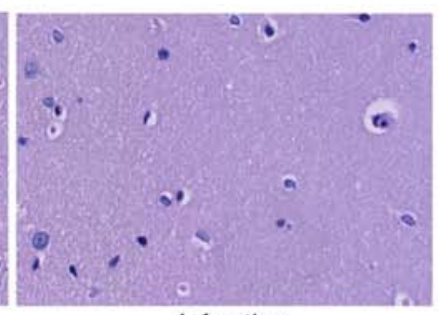

Infarction
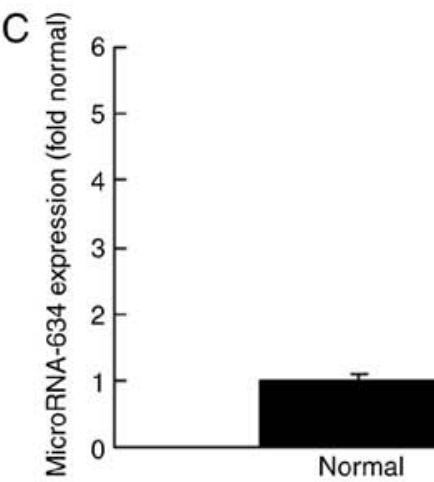

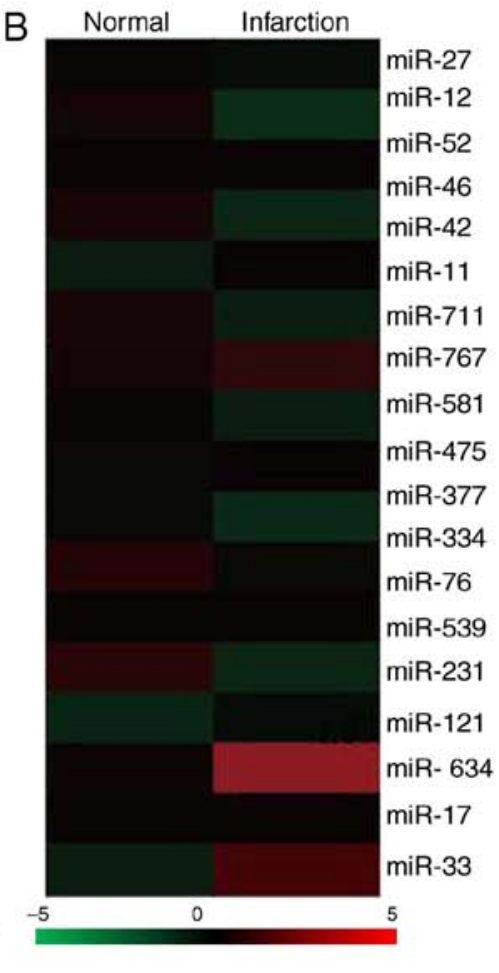

Figure 1. Expression of miRNA-634 in the cerebral infarction rat model. (A) Neurocyte death was apparent in the cerebral infarction group (magnification, $\mathrm{x} 200$ ). (B) Gene chip (green color indicates downregulated miRs and red color indicates upregulated miRs) and (C) reverse transcription-quantitative polymerase chain reaction analyses were performed to analyze the expression levels of miRNA-634. Normal, control rat group; cerebral infarction, cerebral infarction rat group. ${ }^{\# \#} \mathrm{P}<0.01$, vs. control. $\mathrm{miR} / \mathrm{miRNA}$, microRNAs.

significance was determined using one-way analysis of variance with Student's t-test or one-way analysis of variance and Tukey's post hoc test. $\mathrm{P}<0.05$ was considered to indicate a statistically significant difference.

\section{Results}

Expression of miRNA-634 in the cerebral infarction rat model. To determine the expression level of miRNA-634 in the cerebral infarction rat model, RT-qPCR and gene chip analyses were performed to analyze the expression level of miRNA-634. As shown in Fig. 1A, neurocyte death was apparent in the cerebral infarction rat model, compared with the normal group. The expression level of miRNA-634 in the cerebral infarction rat group was increased compared with that in the normal control group (Fig. 1B and C).

Upregulation of the expression of miRNA-634 increases cell apoptosis in vitro. To investigate the effects of mature miRNA-634 on nerve inflammation and apoptosis in cerebral infarction, miRNA-634 mimics were used to increase the expression of miRNA-634. As shown in Fig. 2A-E, the miRNA-634 mimics significantly increased the expression of miRNA-634, inhibited cell proliferation and induced apoptosis in the in vitro model of cerebral infarction, compared with the control negative group. There were also significant increases in the levels of capsase-3, capsase- 8 and capsase- 9 in the in vitro model of cerebral infarction with upregulated miRNA-634, compared with levels in the negative control group (Fig. 2F-H).
Downregulation of the expression of miRNA-634 suppresses cell apoptosis in vitro. The present study also determined the function of the downregulation of miRNA-634 on cell apoptosis in the in vitro model of cerebral infarction. The downregulation of miRNA-634 promoted cell proliferation and inhibited apoptosis in the in vitro model of cerebral infarction, compared with the control negative group (Fig. 3A-E). The downregulation of miRNA-634 decreased the levels of capsase- 3 , capsase- 8 and capsase- 9 in the in vitro model of cerebral infarction with downregulated miRNA-634, compared with levels in the negative control group (Fig. 3F-H).

miRNA-634 regulates the MDM2/p53/Bax pathway in vitro. The present study also aimed to validate the effect on the 53/Bax pathway in the in vitro model of cerebral infarction following miRNA-634 exposure. As shown in Fig. 4A-D, the upregulation of miRNA-634 suppressed the protein expression of MDM2, and induced the protein expression of p53 and Bax in the in vitro model of cerebral infarction, compared with expression in the negative control group. The downregulation of miRNA-634 induced the protein expression of MDM2, and suppressed the protein expression of $\mathrm{p} 53$ and Baxin the in vitro model of cerebral infarction, compared with the expression in the negative control group (Fig. 4E-H).

miRNA-634 regulates the PI3K/Akt pathway in vitro. To determine the mechanism underlying the effect of miRNA-634 on nerve apoptosis of cerebral infarction, the protein expression levels of PI3K and p-Akt were measured using western blot analysis and immunofluorescence. As 

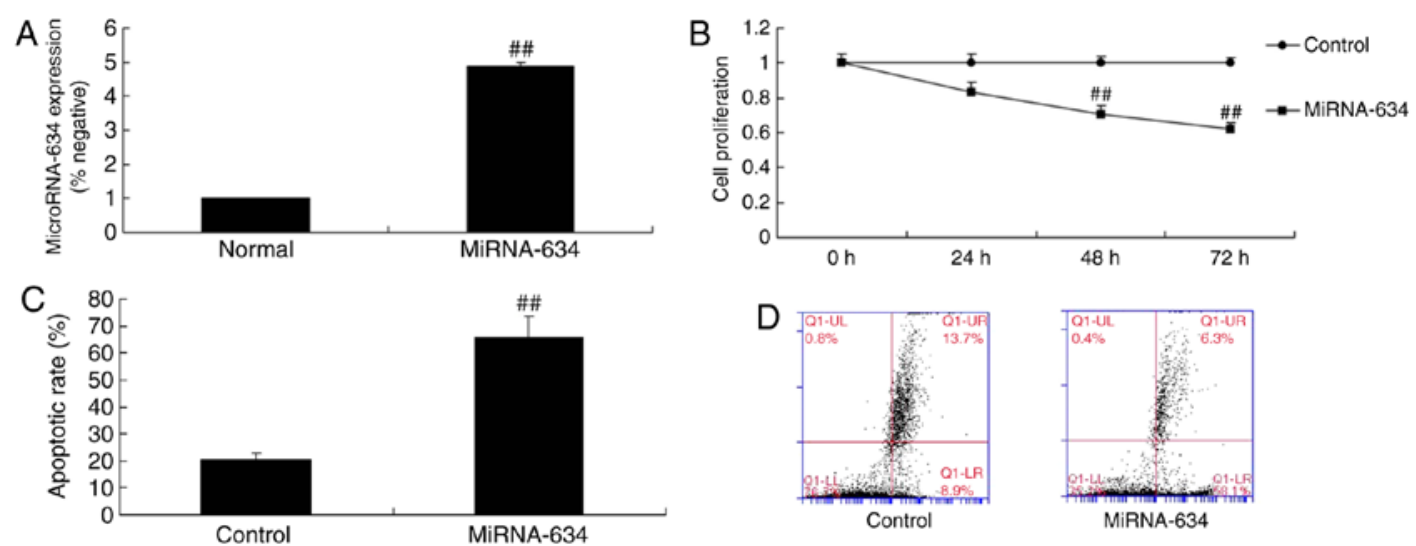

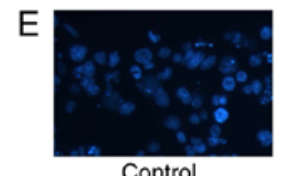

Control

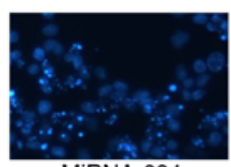

MiRNA-634

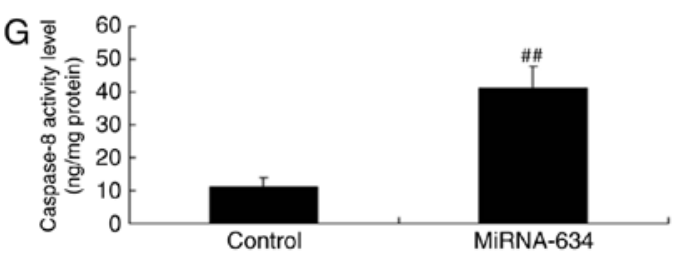

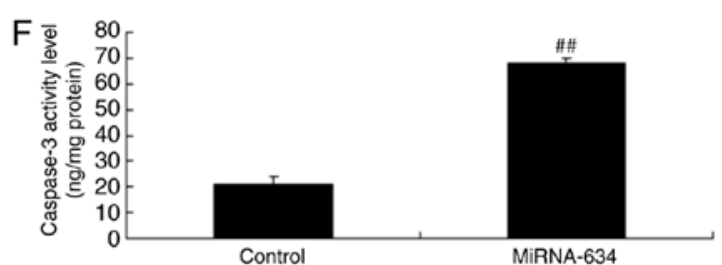

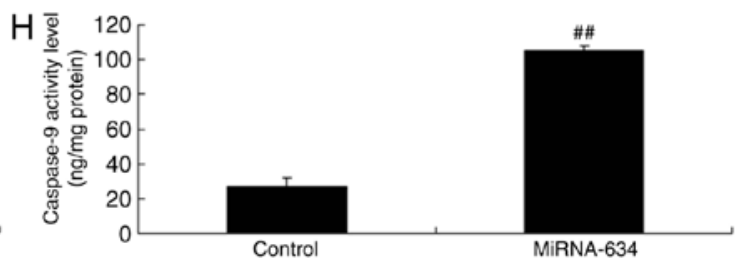

Figure 2. Upregulation of the expression of miRNA-634 increases cell apoptosis in vitro. (A) Expression of miRNA-634; (B) cell proliferation, (C) quantification of cell apoptosis from (D) flow cytometry; (E) DAPI staining (magnification, x100). Activity of (F) caspase-3, (G) caspase-8 and (H) caspase-9 in the in vitro model. Control group, negative control group; miRNA-634, overexpression of miRNA-634 group. ${ }^{\# \#} \mathrm{P}<0.01$, vs. control. miRNA, microRNA.
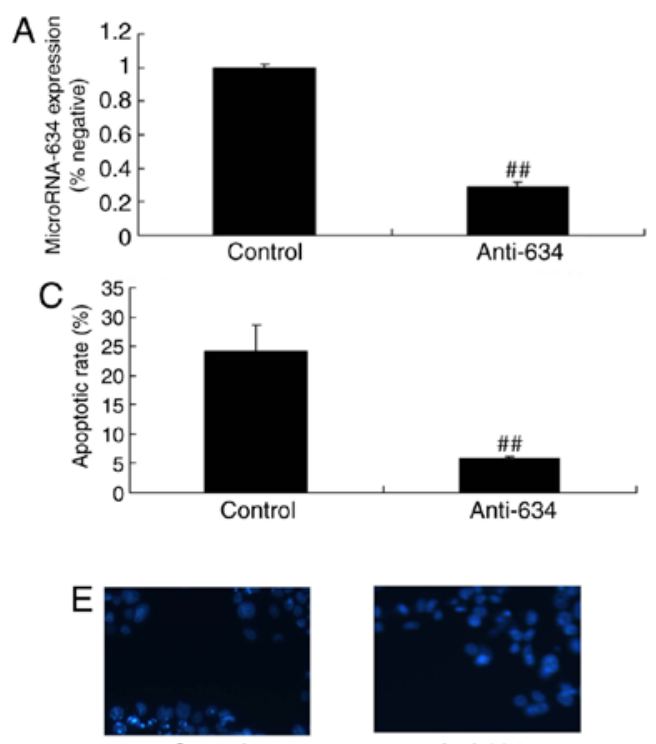

Control

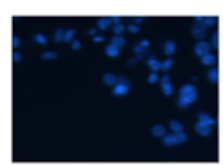

Anti-634

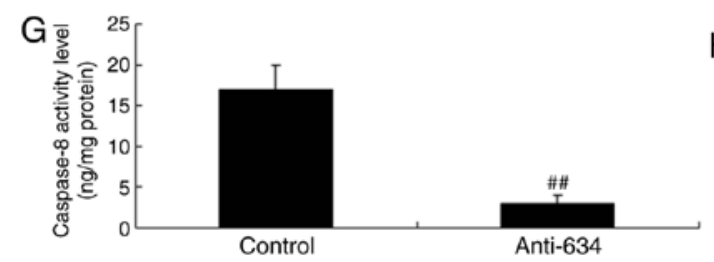

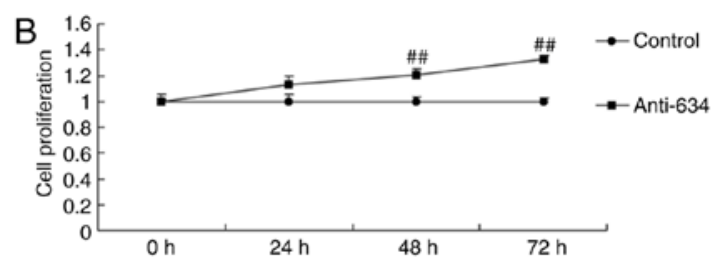
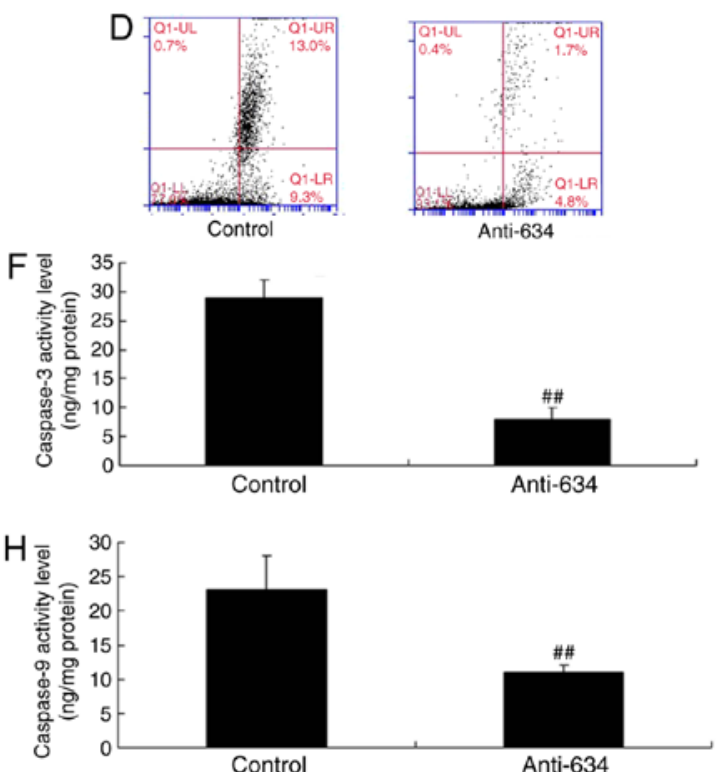

Figure 3. Downregulation of the expression of miRNA-634 suppresses cell apoptosis in vitro. (A) Expression of miRNA-634; (B) cell proliferation; (C) quantification of (D) results of cell apoptosis; (E) DAPI staining (magnification, $x 100$ ); activity levels of (F) caspase-3; (G) caspase-8 and (H) caspase-9 in the in vitro model. Control, negative control group; antii-634, downregulation of miRNA-634 group. ${ }^{\sharp} \mathrm{P}<0.01$, vs. control negative group. miRNA-634, microRNA-634. 

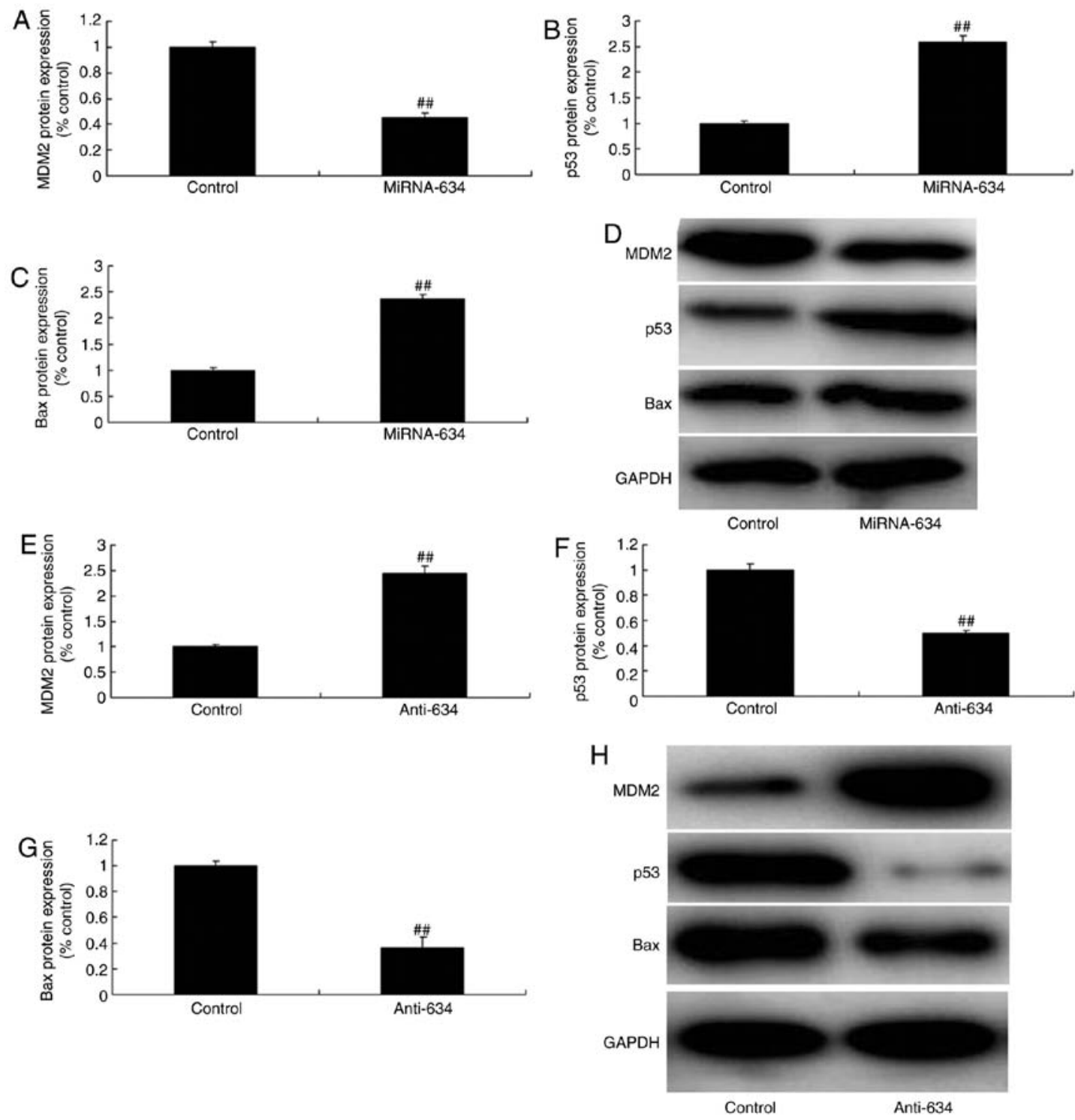

Figure 4. miRNA-634 regulates the MDM2/p53/Bax pathway in vitro. Statistical analysis of the protein expression levels of (A) MDM2, (B) p53 and (C) Bax from (D) western blot analysis in the in vitro model overexpressing miRNA-634. Statistical analysis of protein expression of (E) MDM2, (F) p53 and (G) Bax from $(\mathrm{H})$ western blot analysis in the in vitro model of downregulated miRNA-634. Control, negative control group; miRNA-634, overexpression of miRNA-634 group; anti-634, downregulation of miRNA-634 group. ${ }^{\sharp} \mathrm{P}<0.01$, vs. control. miRNA, microRNA; MDM2, MDM2 proto-oncogene; Bax, B-cell lymphoma 2 -associated $\mathrm{X}$ protein.

the schematic in Fig. 5A shows, the 3' untanslated region of PI3K contains the miRNA-634 seed sites. The results of the immunofluorescence showed that miRNA-634 suppressed the protein expression of $\mathrm{PI} 3 \mathrm{~K}$ in the in vitro model of cerebral infarction, compared with that in the negative control group (Fig. 5B). As shown in Fig. 5C-E, the upregulated expression of miRNA-634 suppressed the protein expression of PI3K and $\mathrm{p}$-Akt in the in vitro model of cerebral infarction, compared with the expression in the negative control group. The downregulation of miRNA-634 induced the protein expression of $\mathrm{PI} 3 \mathrm{~K}$ and $\mathrm{p}$-Akt in the in vitro model of cerebral infarction, compared with the expression in the negative control group (Fig. 5F-H).

Promotion of PI3K reduces the effects of miRNA-634 in vitro. Additionally, the present study assessed the role of PI3K in the effects of microRNA-634 in the in vitro model. As shown in Fig. 6, the PI3K agonist (10 ng/ml of 1,3-dicaffeoylquinic acid) induced the expression of PI3K. The protein expression of $\mathrm{p}$-Akt in the in vitro model of cerebral infarction was also induced by miRNA-634 and the PI3K agonist, compared with that in the miRNA-634 group (Fig. 6A-C). The PI3K agonist induced the protein expression of MDM2, and suppressed the protein expression of p53 and Bax in the in vitro model of cerebral infarction by anti-miRNA-634, compared with the anti-miRNA-634 group (Fig. 6D-G). The PI3K agonist was shown to reduce the effects of miRNA-634 on cell proliferation, apoptosis and caspase-3/8/9 activity levels in the in vitro model of cerebral infarction by miRNA-634, compared with levels in the miRNA-634 group (Fig. 7A-G).

Inhibition of PI3K reduces the effects of anti-miRNA-634 in vitro. To assess the mechanism underlying the effect of miRNA-634 on apoptosis in the in vitro model of cerebral 

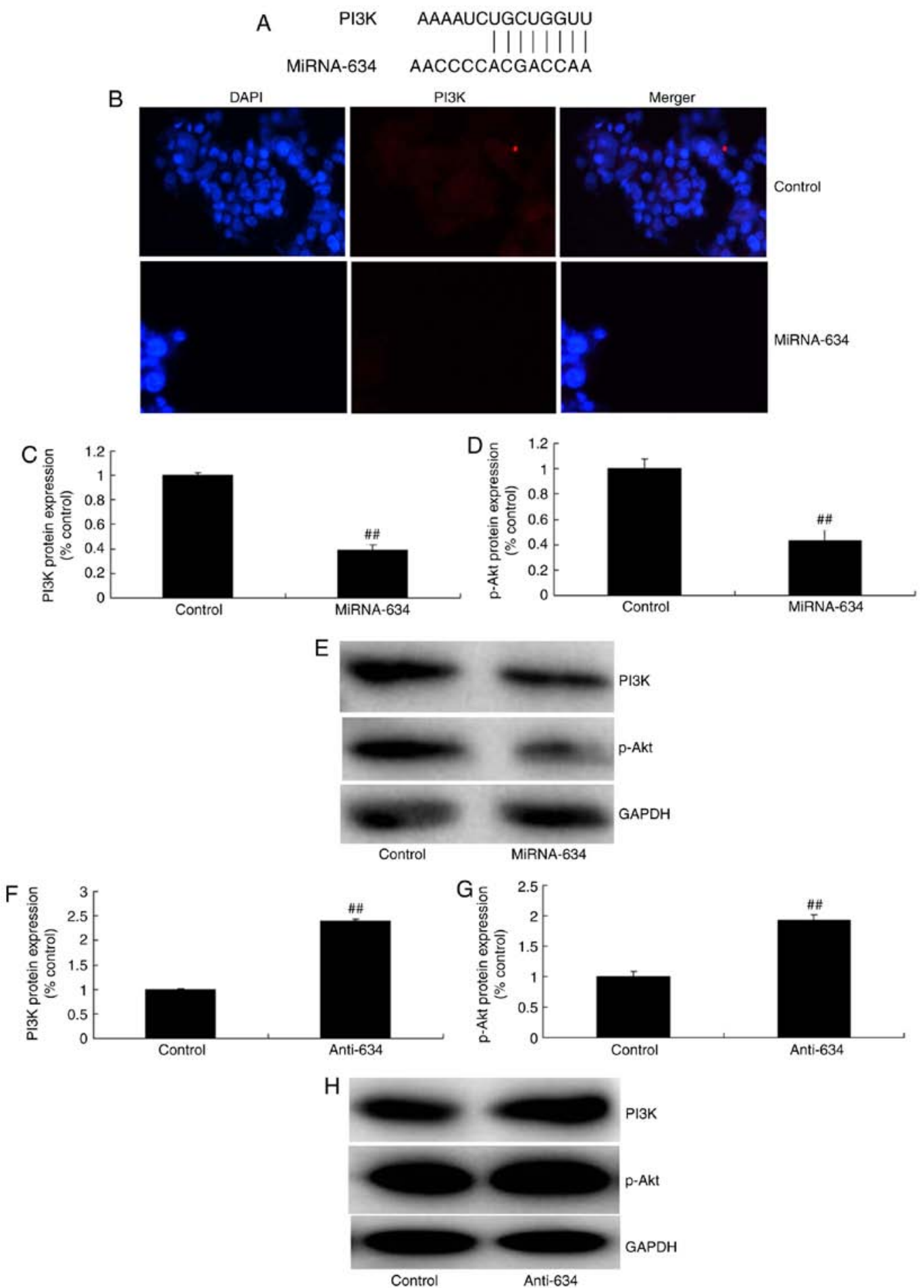

Figure 5. miRNA-634 regulates the PI3K/Akt pathway in vitro. (A) Schematic showing that the 3' untranslated region of PI3K contains the miRNA-634 seed sites. (B) Results of immunofluorescence showed the protein expression of PI3K (magnification, x100). Statistical analysis of a the protein expression of (C) PI3K and (D) p-Akt from (E) western blot analysis in the in vitro model overexpressing miRNA-634. Statistical analysis of a the protein expression of $(\mathrm{F}) \mathrm{PI} 3 \mathrm{~K}$ and $(\mathrm{G}) \mathrm{p}$-Akt from $(\mathrm{H})$ western blot analysis in the in vitro model with downregulated miRNA-634. ${ }^{\# \#} \mathrm{P}<0.01$, vs. control. Control, negative control group; miRNA-634, overexpression of miRNA-634 group; anti-634, downregulated expression of miRNA-634 group. miRNA, microRNA; PI3K, phosphoinositide 3-kinase; p-, phosphorylated.

infarction, the PI3K inhibitor (20 nM of NVP-BAG956), was used to inhibit the protein expression of PI3K. The results of western blot analysis showed that the PI3K inhibitor suppressed the protein expression of PI3K and p-Akt in the in vitro model of cerebral infarction by anti-miRNA-634, compared with the anti-miRNA-634 group (Fig. 8A-C). The PI3K inhibitor suppressed the protein expression of MDM2, and induced the protein expression of $\mathrm{p} 53$ and $\mathrm{Bax}$ in the in vitro model of cerebral infarction by anti-miRNA-634, compared with the anti-miRNA-634 group (Fig. 8D-G). The PI3K inhibitor also reduced the effects of anti-miRNA-634 on cell proliferation, apoptosis and caspase-3/8/9 activity levels in the in vitro model 

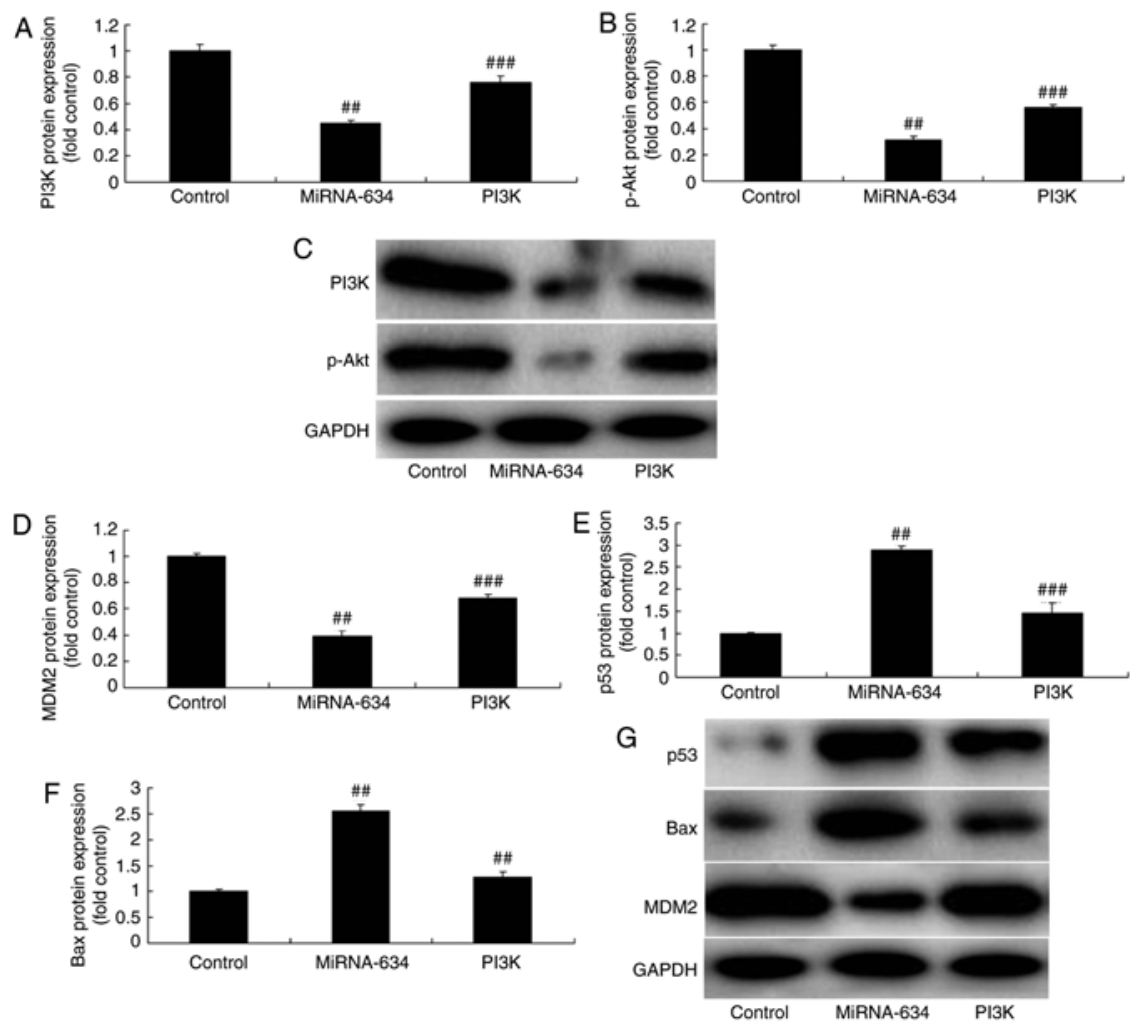

Figure 6. Promotion of PI3K reduces the effects of miRNA-634 on the PI3K/Akt pathway in vitro. Statistical analysis of protein expression levels of (A) PI3K and (B) from (C) western blot analysis. Statistical analysis of protein expression levels of (D) MDM2, (E) p53 and (F) Bax from (G) western blot analysis in the in vitro model. ${ }^{\# \#} \mathrm{P}<0.01$, vs. control; ${ }^{\# \# \#} \mathrm{P}<0.01$, vs. overexpression of miRNA-634 group. Control, negative control group; miRNA-634, overexpression of miRNA-634 group; PI3K, overexpression of miRNA-634 and 1,3-dicaffeoylquinic acid group. miRNA, microRNA; PI3K, phosphoinositide 3-kinase; p-, phosphorylated; MDM2, MDM2 proto-oncogene; Bax, B-cell lymphoma 2-associated X protein.
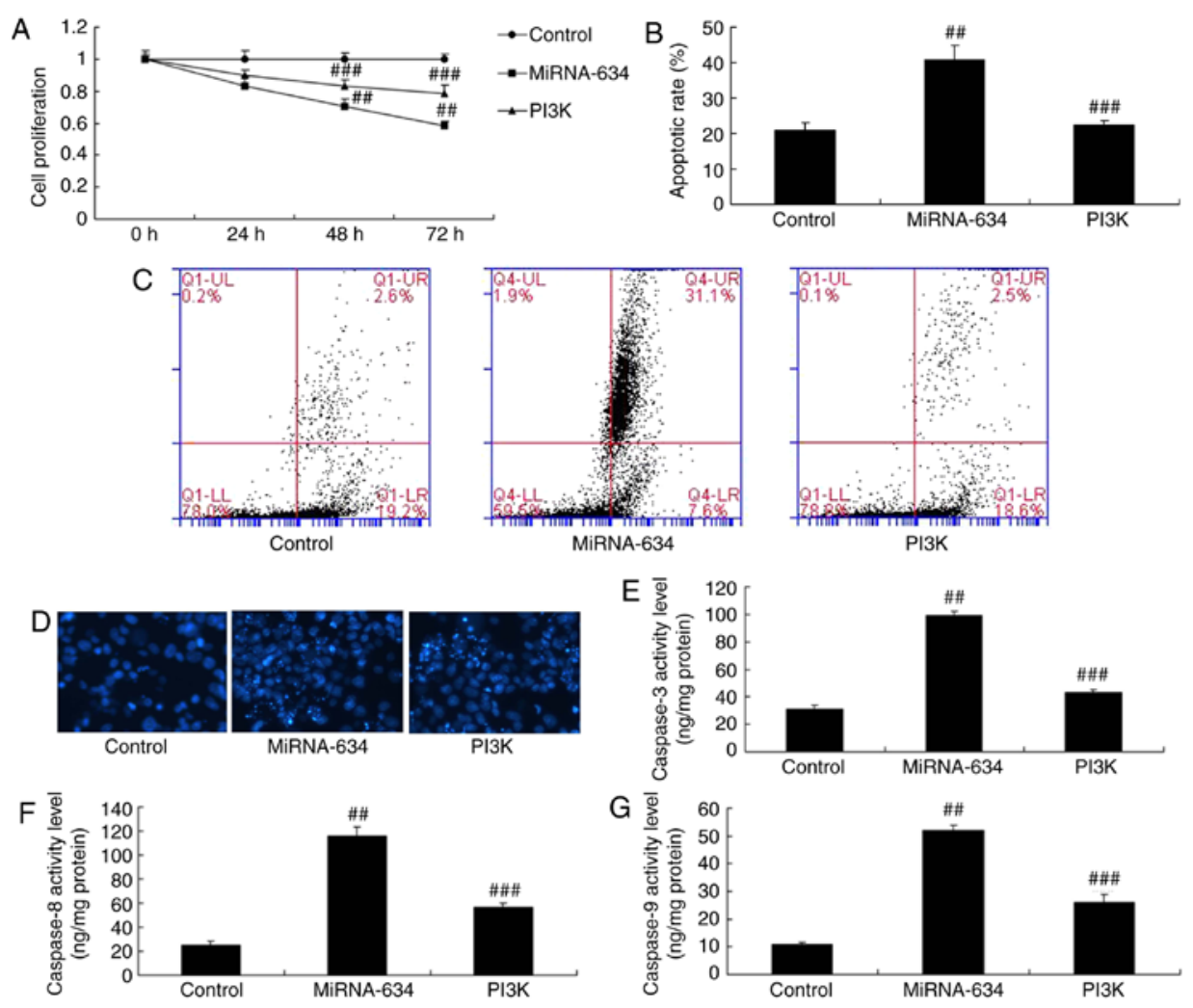

Figure 7. Promotion of PI3K reduces the effects of miRNA-634 on cell apoptosis in vitro. (A) Cell proliferation; (B) quantification of cell apoptosis from (C) results of flow cytometry; (D) DAPI staining (magnification, $x 100$ ); activity levels of (E) caspase-3, (F) caspase-8 and (G) caspase-9 in the in vitro model. ${ }^{\# \prime} \mathrm{P}<0.01$, vs. control; ${ }^{\# \#} \mathrm{P}<0.01$, vs. miRNA-634 group. Control, negative control group; miRNA-634, overexpression of miRNA-634 group; PI3K, overexpression of miRNA-634 and 1,3-dicaffeoylquinic acid group; miRNA, microRNA; PI3K, phosphoinositide 3-kinase. 
A
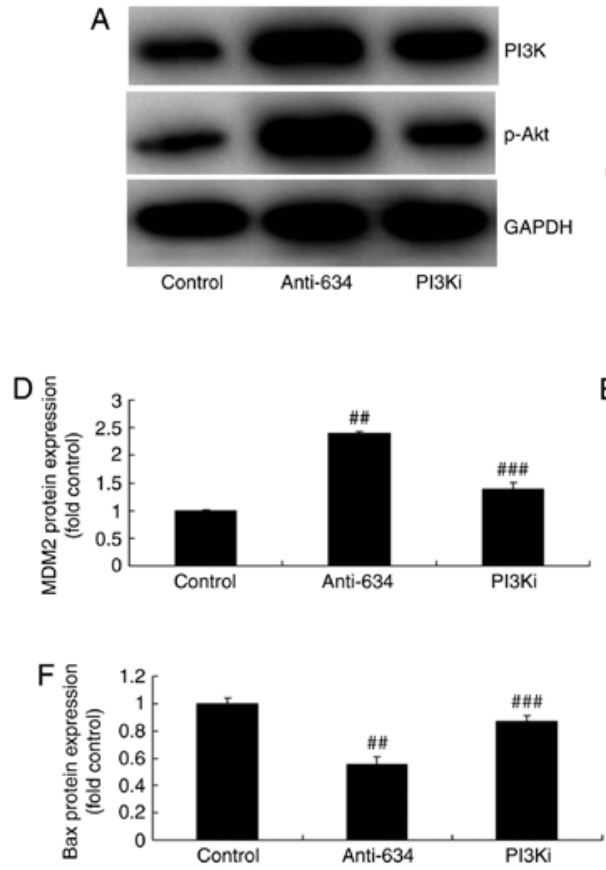
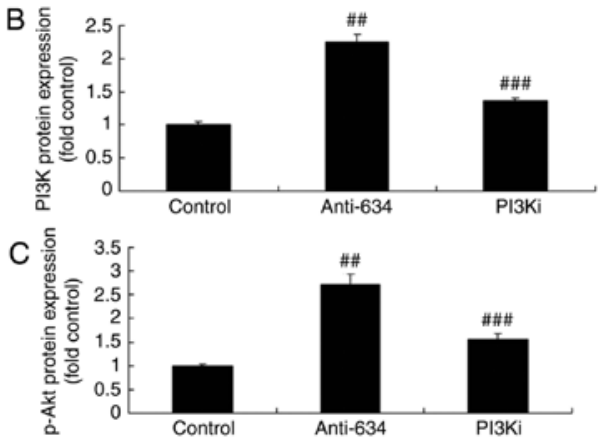

E 음

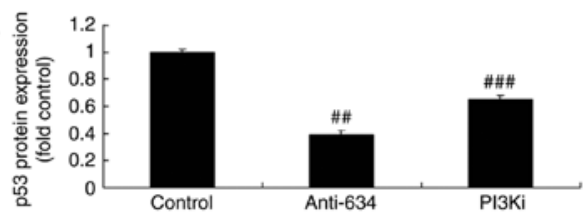

G

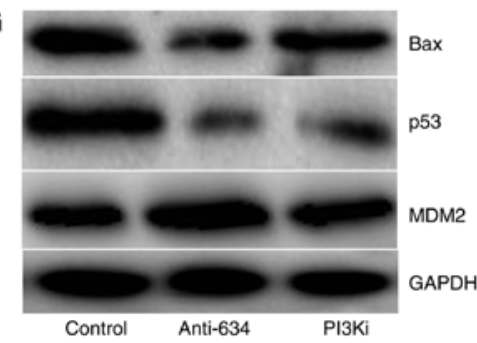

Figure 8. Inhibition of PI3K reduces the effects of anti-miRNA-634 on the PI3K/Akt pathway in vitro. (A) Western blot analysis of the protein expression of (B) PI3K and (C) p-Akt. Statistical analysis of the protein expression of (D) MDM2, (E) p53 and (F) Bax from (G) western blot analysis in the in vitro model. ${ }^{\# \#} \mathrm{P}<0.01$, vs. control; \#\#" $\mathrm{P}<0.01$, vs. downregulation of miRNA-634 group. Control, negative control group; anti-634, downregulation of miRNA-634 group; PI3Ki, downregulation of miRNA-634 and NVP-BAG956 group; miRNA, microRNA; PI3K, phosphoinositide 3-kinase; MDM2, MDM2 proto-oncogene; Bax, B-cell lymphoma 2-associated X protein; p-, phosphorylated.
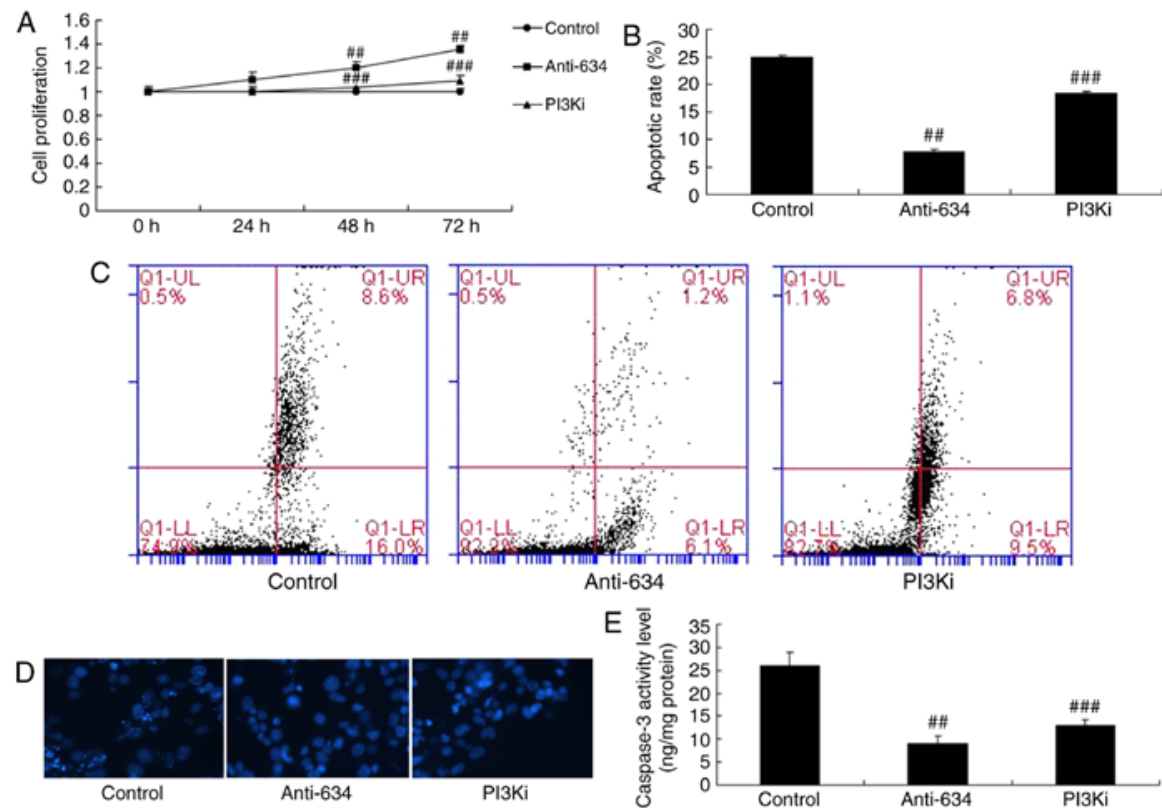

$\mathrm{E}$
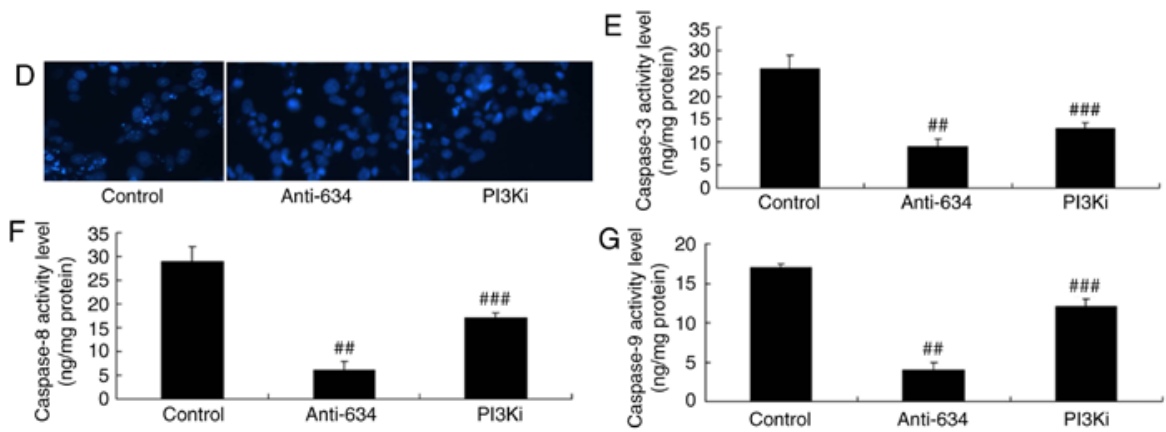

Figure 9. Inhibition of PI3K reduces the effects of anti-miRNA-634 on cell apoptosis in vitro. (A) Cell proliferation; (B) quantification of cell apoptosis from (C) flow cytometry; (D) DAPI staining (magnification, $x 100$ ), activity levels of (E) caspase-3, (F) caspase-8 and (G) caspase-9 in the in vitro model. ${ }^{\# \#} \mathrm{P}<0.01$, vs. control; ${ }^{\# \#} \mathrm{P}<0.01$, vs. downregulation of miRNA-634 group. Control, negative control group; anti-634, downregulation of miRNA-634 group; PI3Ki, downregulation of miRNA-634 and NVP-BAG956 group; miRNA, microRNA; PI3K, phosphoinositide 3-kinase. 


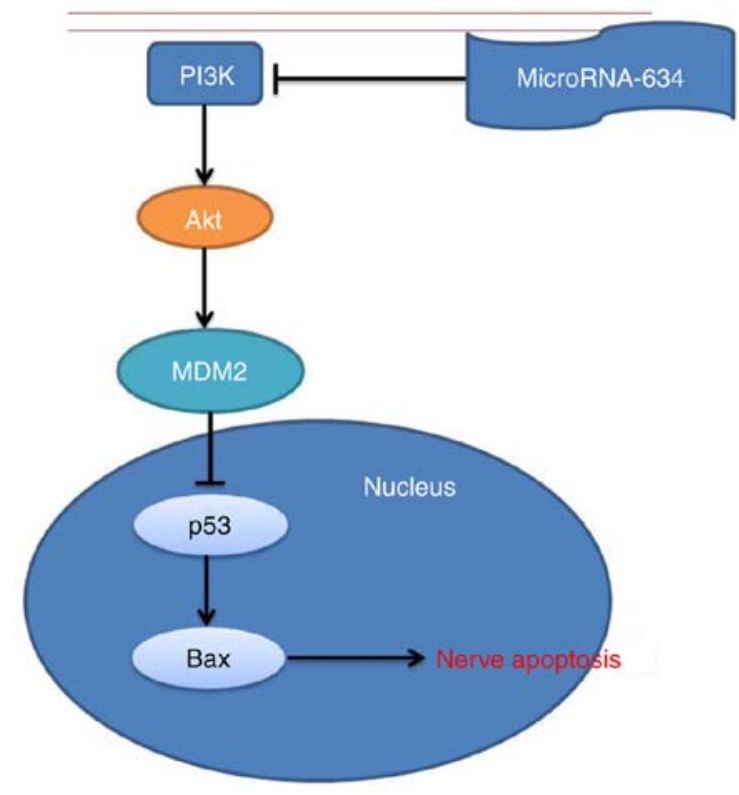

Figure 10. MicroRNA-634 alters nerve apoptosis via the PI3K/Akt pathway in cerebral infarction. PI3K, phosphoinositide 3-kinase; MDM2, MDM2 proto-oncogene; Bax, B-cell lymphoma 2-associated X protein.

of cerebral infarction by anti-miRNA-634, compared with the anti-miRNA-634 group (Fig. 9A-G).

\section{Discussion}

Acute cerebrovascular disease is a common and frequently occurring disease in middle-aged and elderly individuals (11). It is associated with high rates of disability and mortality. The morbidity rate of atherosclerosis has shown an increasing and younger trend year on year with the population aging, and changes to living and social environments. Therefore, it is a serious threat to human health and life (3). In the present study, it was showed that the expression level of miRNA-634 in a cerebral infarction rat model was increased, compared with that in the normal control group. Jeansonne et al (12) showed that the expression of miRNA-634 was upregulated in glioblastoma.

The detection of miRNAs in human tissues is either difficult to achieve or repeatedly used in clinic. It has been found in previous studies that there are stable miRNA molecules in the peripheral blood (13). Additionally, different diseases are associated with different miRNA expression profiles. This finding has led to novel thinking in adopting peripheral blood miRNA for the non-invasive diagnosis of disease (7). It is found that peripheral blood miRNA with specific expression changes can be observed in multiple diseases, including tumors, diabetes, myocardial infarction, Parkinson's disease and Alzheimer's disease (14). In addition, the present study found that miRNA-634 mimics significantly inhibited cell proliferation and induced apoptosis in an in vitro model of cerebral infarction. Cong et al (15) showed that miRNA-634 also decreases cell proliferation and induces apoptosis in cervical cancer cells.

The PI3K/Akt signal transduction pathway is a pro-survival signal. Its activation is important in ischemic-hypoxic neuronal injury, and it has attracted increasing attention (16). The PI3K/Akt signal transduction pathway is an important pathway for the intracellular transduction of membrane receptor signals. It is key in maintaining cell survival and inhibiting cell apoptosis (17). It can affect the activation of effector molecules, including downstream apoptosis-related protein and cell cycle regulatory protein (17). Consequently, it is important in inhibiting apoptosis and promoting proliferation in cells (9). PI3K/Akt has a definite anti-apoptotic protective effect on the brain in cerebral ischemia. Inhibiting such a pathway can aggravate cerebral ischemia-induced nerve cell apoptosis (9). The findings of the present study suggested that downregulation of the expression of miRNA-634 significantly suppressed the protein expression of PI3K and p-Akt in the in vitro model of cerebral infarction. Cui et al (18) suggested that the overexpression of miRNA-634 suppresses survival and matrix synthesis by targeting PIK3 regulatory subunit 1 in human osteoarthritic chondrocytes.

Caspase-3 is the most important apoptotic protease during apoptosis. Its activation is dependent on the release of cytochrome $c$ (19). The Bcl-2 and Bax genes of the Bcl-2 family are the most important regulatory genes involved in cell apoptosis known at present. They can mediate the release of substances, including cytochrome $c$, through the mitochondrial pathway (20). A number of previous studies have found that $\mathrm{Bcl}-2$ and $\mathrm{Bax}$ can serve as the upstream regulatory mechanism of caspase- 3 and are involved in regulating the activity of caspase-3 (20). In addition, they can be treated as the direct substrate of caspase- 3 to act on the downstream caspase-3. The two correlate with and restrain each other during cell apoptosis transduction (21). In the present study, it was shown that the inhibition of PI3K increased the effect of the downregulation of miRNA-634 on cell apoptosis and capsase-3/Bax protein expression in the in vitro model of cerebral infarction.

In conclusion, the findings of the present study suggested that the plasma miRNA-634 adjusted nerve apoptosis in cerebral infarction through the MDM2/p53/Bax pathway by PI3K/Akt (Fig. 10). miRNA-634 may be used as a potential therapeutic target in the treatment of cerebral infarction for clinical use in the future.

\section{Acknowledgements}

Not applicable.

\section{Funding}

No funding was received.

\section{Availability of data and materials}

The analysed data sets generated during the study are available from the corresponding author on reasonable request.

\section{Authors' contributions}

QS designed the study; YC, WH, SL, ZY, QW and XL performed the experiments; QS and YC analyzed the data; and QS wrote the manuscript. 


\section{Ethics approval and consent to participate}

The study was approved by the Animal Experimental Ethics Committee of Tangshan, Worker Hospital (Tangshan, China).

\section{Patient consent for publication}

Not applicable.

\section{Competing interests}

The authors declare that they have no competing interests.

\section{References}

1. Afshari D, Moradian N, Nasiri F, Razazian N, Bostani A and Sariaslani P: The efficacy and safety of low-molecular-weight heparin and unfractionated heparin in the treatment of cerebral venous sinus thrombosis. Neurosciences (Riyadh) 20: 357-361, 2015.

2. Berge J, Blanco P, Rooryck C, Boursier R, Marnat G, Gariel F, Wavasseur T, Desal $\mathrm{H}$ and Dousset V: Understanding flow patterns and inflammatory status in intracranial aneurysms: Towards a personalized medicine. J Neuroradiol 43: 141-147, 2016.

3. Zhou Y, Yang PF, Fang YB, Xu Y, Hong B, Zhao WY, Li Q, Zhao R, Huang QH and Liu JM: Parent artery reconstruction for large or giant cerebral aneurysms using a Tubridge flow diverter (PARAT): Study protocol for a multicenter, randomized, controlled clinical trial. BMC Neurol 14: 97, 2014.

4. Cebral J, Ollikainen E, Chung BJ, Mut F, Sippola V, Jahromi BJ, Tulamo R, Hernesniemi J, Niemelä M, Robertson A and Frösen J: Flow conditions in the intracranial aneurysm lumen are associated with inflammation and degenerative changes of the aneurysm wall. AJNR Am J Neuroradiol 38: 119-126, 2017.

5. Yuan M, Zhan Q, Duan X, Song B, Zeng S, Chen X, Yang Q and Xia J: A functional polymorphism at miR-491-5p binding site in the 3'-UTR of MMP-9 gene confers increased risk for atherosclerotic cerebral infarction in a Chinese population. Atherosclerosis 226: 447-452, 2013.

6. WangC,Pan Y,ChengB,ChenJandBaiB:Identification of conserved and novel microRNAs in cerebral ischemia-reperfusion injury of rat using deep sequencing. J Mol Neurosci 54: 671-683, 2014.

7. Tian C, Li Z, Yang Z, Huang Q, Liu J and Hong B: Plasma microRNA-16 is a biomarker for diagnosis, stratification, and prognosis of hyperacute cerebral infarction. PLoS One 11: e0166688, 2016

8. Peng B, Guo QL, He ZJ, Ye Z, Yuan YJ, Wang N and Zhou J: Remote ischemic postconditioning protects the brain from global cerebral ischemia/reperfusion injury by up-regulating endothelial nitric oxide synthase through the PI3K/Akt pathway. Brain Res 1445: 92-102, 2012.
9. Liu XY, Zhou XY, Hou JC, Zhu H, Wang Z, Liu JX and Zheng YQ: Ginsenoside Rd promotes neurogenesis in rat brain after transient focal cerebral ischemia via activation of PI3K/Akt pathway. Acta Pharmacol Sin 36: 421-428, 2015.

10. Livak KJ and Schmittgen TD: Analysis of relative gene expression data using real-time quantitative PCR and the 2(-Delta Delta C(T)) Method. Methods 25: 402-408, 2001.

11. Iveson T, Donehower RC, Davidenko I, Tjulandin S, Deptala A, Harrison M, Nirni S, Lakshmaiah K, Thomas A, Jiang Y, et al: Rilotumumab in combination with epirubicin, cisplatin, and capecitabine as first-line treatment for gastric or oesophagogastric junction adenocarcinoma: An open-label, dose de-escalation phase $1 \mathrm{~b}$ study and a double-blind, randomised phase 2 study. Lancet Oncol 15: 1007-1018, 2014.

12. Jeansonne D, Pacifici M, Lassak A, Reiss K, Russo G, Zabaleta J and Peruzzil F: Differential effects of microRNAs on glioblastoma growth and migration. Genes (Basel) 4: 46-64, 2013.

13. Liu XS, Chopp M, Zhang RL and Zhang ZG: MicroRNAs in cerebral ischemia-induced neurogenesis. J Neuropathol Exp Neurol 72: 718-722, 2013.

14. Kortvelyessy P, Huchtemann T, Heinze HJ and Bittner DM: Progranulin and its related microRNAs after status epilepticus: Possible mechanisms of neuroprotection. Int J Mol Sci 18: 2017.

15. Cong J, Liu R, Wang X, Jiang H and Zhang Y: miR-634 decreases cell proliferation and induces apoptosis by targeting mTOR signaling pathway in cervical cancer cells. Artif Cells Nanomed Biotechnol 44: 1694-1701, 2016.

16. Ji K, Xue L, Cheng J and Bai Y: Preconditioning of H2S inhalation protects against cerebral ischemia/reperfusion injury by induction of HSP70 through PI3K/Akt/Nrf2 pathway. Brain Res Bull 121: 68-74, 2016

17. Liang K, Ye Y, Wang Y, Zhang J and Li C: Formononetin mediates neuroprotection against cerebral ischemia/reperfusion in rats via downregulation of the $\mathrm{Bax} / \mathrm{Bcl}-2$ ratio and upregulation PI3K/Akt signaling pathway. J Neurol Sci 344: 100-104, 2014.

18. Cui X, Wang S, Cai H, Lin Y, Zheng X, Zhang B and Xia C: Overexpression of microRNA-634 suppresses survival and matrix synthesis of human osteoarthritis chondrocytes by targeting PIK3R1. Sci Rep 6: 23117, 2016.

19. Wang X, Luo Y, Sun H, Feng J, Ma S, Liu J and Huang B: Dynamic expression changes of Bcl-2, Caspase-3 and Hsp70 in middle cerebral artery occlusion rats. Brain Inj 29: 93-97, 2015.

20. Zhang HR, Peng JH, Zhu GY and Xu RX: Neuroprotective effects of Bcl-2 overexpression on nerve cells of rats with acute cerebral infarction. Genet Mol Res 14: 7696-7703, 2015.

21. Cheng CY, Tang NY, Kao ST and Hsieh CL: Ferulic acid administered at various time points protects against cerebral infarction by activating p38 MAPK/p90RSK/CREB/Bcl-2 anti-apoptotic signaling in the subacute phase of cerebral ischemia-reperfusion injury in rats. PLoS One 11: e0155748, 2016. 